\title{
Background field method in the gradient flow
}

\author{
Hiroshi Suzuki ${ }^{* \dagger}$ \\ Department of Physics, Kyushu University, 744 Motooka, Nishi-ku, Fukuoka, 819-0395, Japan \\ E-mail: hsuzukiephys.kvushu-u.ac.ip
}

The Yang-Mills gradient flow and its extension to the fermion field provide a very general method to obtain renormalized observables in gauge theory. The method is applicable also with nonperturbative regularization such as lattice. The gradient flow thus offers useful probes to study non-perturbative dynamics of gauge theory. In this work, aiming at possible simplification in perturbative calculations associated with the gradient flow, a modification of the gauge-fixed version of the flow equation, which preserves gauge covariance under the background gauge transformation, is proposed. This formulation allows for example a very quick one-loop calculation of the small flow time expansion of a composite operator that is relevant to the construction of a lattice energy-momentum tensor. Some details of the calculation, which have not been given elsewhere, are presented.

The 33rd International Symposium on Lattice Field Theory

14 -18 July 2015

Kobe International Conference Center, Kobe, Japan*

\footnotetext{
*Speaker.

${ }^{\dagger}$ The work of H. S. is supported in part by Grant-in-Aid for Scientific Research 23540330.

$¥$ Address after October 1st.
} 


\section{Introduction}

The Yang-Mills gradient flow [ $[\mathbf{l}, \mathbb{2}]$ and its extension to the fermion field [ [ $]$ ] provide a very general method to obtain renormalized observables in gauge theory. The method is applicable also with non-perturbative regularization such as lattice. The gradient flow thus offers useful probes to study non-perturbative dynamics of gauge theory. See Ref. [团] for a recent review.

As noted in Ref. [四, for perturbative consideration of the gradient flow, it is useful to introduce a "gauge fixing term" to the flow equation that breaks gauge covariance. This gauge fixing term gives rise to a Gaussian damping factor also for gauge degrees of freedom which ensures a good convergence property of momentum integrals; this then facilitates perturbative consideration of the gradient flow such as the proof of its renormalizability [2], []].

In this work, aiming at possible simplification in perturbative calculations associated with the gradient flow, a modification of (the gauge-fixed version of) the flow equation of Ref. [W] is proposed. Our proposal is

$$
\partial_{t} B_{\mu}(t, x)=D_{v} G_{v \mu}(t, x)+\alpha_{0} D_{\mu} \hat{D}_{v} b_{v}(t, x), \quad B_{\mu}(t=0, x)=A_{\mu}(x),
$$

where $t>0$ is the flow time which parametrizes the flow of the gauge field $B_{\mu}(t, x) ; D_{v}$ and $G_{v \mu}$ stand for the covariant derivative and field strength of the flowed gauge field, respectively. The term being proportional to $\alpha_{0}$ is the "gauge fixing term" mentioned above. To define this term, we decompose the gauge fields into the background and quantum parts as $A_{\mu}(x)=\hat{A}_{\mu}(x)+a_{\mu}(x)$ and, correspondingly, $B_{\mu}(t, x)=\hat{B}_{\mu}(t, x)+b_{\mu}(t, x)$. Then in Eq. (IL. $)$ ),

$$
\hat{D}_{\mu}=\partial_{\mu}+\left[\hat{B}_{\mu}, \cdot\right]
$$

is the covariant derivatives with respect to the background field $\hat{B}_{\mu}(t, x)$. The idea is that, as the

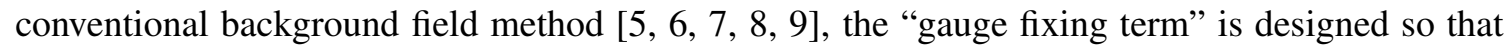
covariance under the background gauge transformation is preserved; the quantum fields transform as the adjoint representation under the background gauge transformation. In addition to Eq. (ID. $)$ ), we postulate that the background field obeys its own flow equation:

$$
\partial_{t} \hat{B}_{\mu}(t, x)=\hat{D}_{v} \hat{G}_{v \mu}(t, x), \quad \hat{B}_{\mu}(t=0, x)=\hat{A}_{\mu}(x),
$$

where $\hat{G}_{v \mu}(t, x)$ is the field strength of the background field $\hat{B}_{\mu}(t, x)$.

Since the present study is already published in Ref. [ए]], in these proceedings, I will reproduce some materials which were not explicitly given in Ref. [س]]. In particular, since the most interesting result obtained in Ref. [U]] is a one-loop calculation of the small flow time expansion of the composite operator $G_{\mu \rho}^{a}(t, x) G_{v \rho}^{a}(t, x)$, which is relevant to the construction of a lattice energy-

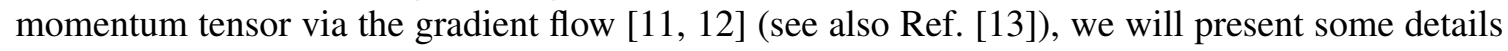
of the calculation ${ }^{1}$ which were omitted in Ref. [ए]]; ; we do not treat the fermion flow in the present article. In this way, I hope that the present article becomes complementary to Ref. [ए]]. Our notational convention is identical to that of Ref. [LU]; in particular, generators of the gauge group are normalized as $\operatorname{tr}\left(T^{a} T^{b}\right)=(-1 / 2) \delta^{a b}$.

\footnotetext{
${ }^{1}$ Our calculational scheme has been originally inspired by the calculation in Ref. [प]य].
} 


\section{Small flow time expansion relevant to the energy-momentum tensor}

The initial condition in the flow equation $(\mathbb{L}), A_{\mu}(x)$, is subject of the functional integral with the Boltzmann weight, specified by the Yang-Mills and background-gauge-fixing actions:

$$
S+S_{\mathrm{gf}}=-\frac{1}{2 g_{0}^{2}} \int d^{D} x \operatorname{tr}\left[F_{\mu v}(x) F_{\mu v}(x)\right]-\frac{\lambda_{0}}{g_{0}^{2}} \int d^{D} x \operatorname{tr}\left[\hat{D}_{\mu} a_{\mu}(x) \hat{D}_{v} a_{v}(x)\right],
$$

here $D \equiv 4-2 \varepsilon$ is the spacetime dimension. The Faddeev-Popov ghost action corresponding to this background gauge fixing is given by

$$
S_{c \bar{c}}=\frac{2}{g_{0}^{2}} \int d^{D} x \operatorname{tr}\left[\bar{c}(x) \hat{D}_{\mu} D_{\mu} c(x)\right] .
$$

From these, the tree-level propagators in the presence of the background field are given by, for the Feynman gauge $\lambda_{0}=1$,

$$
\left\langle a_{\mu}^{a}(x) a_{v}^{b}(y)\right\rangle_{0}=-g_{0}^{2}\left(\frac{1}{\hat{\Delta}_{x}}\right)_{\mu v}^{a b} \delta(x-y), \quad\left\langle c^{a}(x) \bar{c}^{b}(y)\right\rangle_{0}=-g_{0}^{2}\left(\frac{1}{\hat{\mathscr{D}}_{x}^{2}}\right)^{a b} \delta(x-y),
$$

where

$$
\hat{\mathscr{D}}_{\mu}^{a b} \equiv \delta^{a b} \partial_{\mu}+\hat{A}_{\mu}^{c}(x) f^{a c b}
$$

is the background covariant derivative in the adjoint representation; $\left(\hat{\mathscr{D}}^{2}\right)^{a b}=\hat{\mathscr{D}}_{\mu}^{a c} \hat{\mathscr{D}}_{\mu}^{c b}$ and

$$
\hat{\Delta}_{\mu \nu}^{a b}=\left(\hat{\mathscr{D}}^{2}\right)^{a b} \boldsymbol{\delta}_{\mu \nu}+2 \hat{\mathscr{F}}_{\mu \nu}^{a b}, \quad \hat{\mathscr{F}}_{\mu \nu}^{a b}(x) \equiv \hat{F}_{\mu \nu}^{c}(x) f^{a c b} .
$$

In what follows, as Ref. [미], we assume that the background field $\hat{A}_{\mu}(x)$ obeys the YangMills equation of motion: $\hat{D}_{v} \hat{F}_{v \mu}(x)=0$. Eq. (ㄸ.3) then implies that the background gauge field does not flow, $\hat{B}(t, x)=\hat{A}(x)$. This assumption considerably simplifies all the expressions and, in particular, the tree-level propagator of the flowed quantum field in the presence of the background field, for the "Feynman gauge" $\alpha_{0}=1,{ }^{2}$ is given by [ए]]

$$
\left\langle b_{\mu}^{a}(t, x) b_{v}^{b}(s, y)\right\rangle_{0}=-g_{0}^{2}\left[e^{(t+s) \hat{\Delta}_{x}} \frac{1}{\hat{\Delta}_{x}}\right]_{\mu \nu}^{a b} \delta(x-y) .
$$

Now, for the construction of a lattice energy-momentum tensor in Refs. [ए], प2], one has to find the coefficients $\zeta_{11}(t)$ and $\zeta_{12}(t)$ in the small flow time expansion []] of the form,

$$
\begin{aligned}
& G_{\mu \rho}^{a}(t, x) G_{v \rho}^{a}(t, x) \\
& \stackrel{t \rightarrow 0}{\sim}\left\langle G_{\mu \rho}^{a}(t, x) G_{v \rho}^{a}(t, x)\right\rangle+\zeta_{11}(t) F_{\mu \rho}^{a}(x) F_{v \rho}^{a}(x)+\zeta_{12}(t) \delta_{\mu v} F_{\rho \sigma}^{a}(x) F_{\rho \sigma}^{a}(x)+O(t) .
\end{aligned}
$$

For our background-quantum decomposition,

$$
\begin{aligned}
G_{\mu \rho}^{a} G_{v \rho}^{a}= & \hat{F}_{\mu \rho}^{a} \hat{F}_{v \rho}^{a}+\hat{F}_{\mu \rho}^{a}\left(\hat{D}_{v} b_{\rho}-\hat{D}_{\rho} b_{v}\right)^{a}+\left(\hat{D}_{\mu} b_{\rho}-\hat{D}_{\rho} b_{\mu}\right)^{a} \hat{F}_{v \rho}^{a} \\
& +\left(\hat{D}_{\mu} b_{\rho}-\hat{D}_{\rho} b_{\mu}\right)^{a}\left(\hat{D}_{v} b_{\rho}-\hat{D}_{\rho} b_{v}\right)^{a}+\hat{F}_{\mu \rho}^{a}\left[b_{v}, b_{\rho}\right]^{a}+\left[b_{\mu}, b_{\rho}\right]^{a} \hat{F}_{v \rho}^{a}+O\left(b^{3}\right),
\end{aligned}
$$

${ }^{2}$ It can be shown that any gauge invariant quantity that does not contain the flow time derivative is independent of $\alpha_{0}$ [ए]]. 
where we have used $\hat{B}(t, x)=\hat{A}(x)$. A similar expansion holds also for $F_{\mu \rho}^{a} F_{v \rho}^{a}$. Thus, in the tree-level, in which the quantum fields are treated as zero, we have

$$
\left\langle G_{\mu \rho}^{a}(t, x) G_{v \rho}^{a}(t, x)\right\rangle=\hat{F}_{\mu \rho}^{a}(x) \hat{F}_{v \rho}^{a}(x), \quad\left\langle F_{\mu \rho}^{a}(x) F_{v \rho}^{a}(x)\right\rangle=\hat{F}_{\mu \rho}^{a}(x) \hat{F}_{v \rho}^{a}(x),
$$

and from these, $G_{\mu \rho}^{a}(t, x) G_{v \rho}^{a}(t, x) \stackrel{t \rightarrow 0}{\sim} F_{\mu \rho}^{a}(x) F_{v \rho}^{a}(x)+O(t)$ in the tree level.

For the one-loop calculation, as noted in Refs. [피], it is convenient to consider the correlation function

$$
\left\langle G_{\mu \rho}^{a}(t, x) G_{v \rho}^{a}(t, x)-F_{\mu \rho}^{a}(x) F_{v \rho}^{a}(x)\right\rangle,
$$

because possible infrared divergences are cancelled out in this difference. Noting Eq. ( $\mathbb{2 . 8}$ ), we first consider the tadpole contribution to Eq. (2.]

$$
\hat{F}_{\mu \rho}^{a}\left(\hat{D}_{v}\left\langle b_{\rho}-a_{\rho}\right\rangle-\hat{D}_{\rho}\left\langle b_{v}-a_{v}\right\rangle\right)^{a}+\left(\hat{D}_{\mu}\left\langle b_{\rho}-a_{\rho}\right\rangle-\hat{D}_{\rho}\left\langle b_{\mu}-a_{\mu}\right\rangle\right)^{a} \hat{F}_{v \rho}^{a} .
$$

For the one-point function $\left\langle b_{\mu}^{a}(t, x)-a_{\mu}^{a}(x)\right\rangle$ in the presence of the background field, there are two types of one-loop diagrams. The first type is tadpole diagrams containing the vertex at the vanishing flow time. The contribution of this type is

$$
\begin{aligned}
\left\langle b_{\mu}^{a}(t, x)-a_{\mu}^{a}(x)\right\rangle=g_{0}^{2} & {\left[\left(e^{t \hat{\Delta}_{x}}-1\right) \frac{1}{\hat{\Delta}_{x}}\right]_{\mu \nu}^{a b} f^{b c d} } \\
& \times \lim _{y \rightarrow x}\left[-2\left(\frac{1}{\hat{\Delta}_{x}}\right)_{\rho v}^{c e} \hat{\mathscr{D}}_{x \rho}^{e d}+\left(\frac{1}{\hat{\Delta}_{x}}\right)_{\rho \rho}^{c e} \hat{\mathscr{D}}_{x v}^{e d}+\left(\frac{1}{\hat{\Delta}_{x}}-\frac{1}{\hat{\mathscr{D}}_{x}^{2}}\right)_{v \rho}^{c e} \hat{\mathscr{D}}_{x \rho}^{e d}\right] \delta(x-y),
\end{aligned}
$$

where the last term is the contribution of the ghost loop. In this expression, the flow time $t$ is contained only in the first factor and this is obviously $O(t)$. Thus, this first type does not contribute to the $O\left(t^{0}\right)$ terms in Eq. (‥Z).

The second type of diagram is the contribution of the vertex at non-zero flow time (flow vertex):

$$
\left\langle b_{\mu}^{a}(t, x)\right\rangle=g_{0}^{2} \int_{0}^{t} d s\left[e^{(t-s) \hat{\Delta}_{x}}\right]_{\mu v}^{a b} f^{b c d} \lim _{y \rightarrow x}\left[2\left(e^{2 s \hat{\Delta}_{x}} \frac{1}{\hat{\Delta}_{x}}\right)_{\rho v}^{c e} \hat{\mathscr{D}}_{x \rho}^{e d}-\left(e^{2 s \hat{\Delta}_{x}} \frac{1}{\hat{\Delta}_{x}}\right)_{\rho \rho}^{c e} \hat{\mathscr{D}}_{x v}^{e d}\right] \delta(x-y) .
$$

To study the $t \rightarrow 0$ behavior of this, we set $\delta(x-y)=\int \frac{d^{D} p}{(2 \pi)^{D}} e^{i p x} e^{-i p y}$, and moves the plain wave $e^{i p x}$ to the most left-hand side under the $\operatorname{limit}_{\lim } \rightarrow x$ as the Fujikawa method [ए6, [प]]. For this, we note

$$
\hat{\mathscr{D}}_{\mu} e^{i p x}=e^{i p x}\left(i p_{\mu}+\hat{\mathscr{D}}_{\mu}\right),
$$

and rescale the integration variable as $p_{\mu} \rightarrow p_{\mu} / \sqrt{s}$. Then, Eq. ([2.13]) becomes

$$
\begin{aligned}
\left\langle b_{\mu}^{a}(t, x)\right\rangle=g_{0}^{2} \int_{0}^{t} d s\left[e^{(t-s) \hat{\Delta}_{x}}\right]_{\mu v}^{a b} f^{b c d} s^{-D / 2+1 / 2} \int \frac{d^{D} p}{(2 \pi)^{D}} e^{-2 p^{2}} & \\
\times & {\left[2\left(e^{4 i \sqrt{s} p \cdot \hat{\mathscr{D}}+2 s \hat{\Delta}} \frac{1}{-p^{2}+2 i \sqrt{s} p \cdot \hat{\mathscr{D}}+s \hat{\Delta}}\right)_{\rho v}^{c e}\left(i p_{\rho}+\hat{\mathscr{D}}_{\rho}\right)^{e d}\right.} \\
& \left.\quad-\left(e^{4 i \sqrt{s} p \cdot \hat{\mathscr{D}}+2 s \hat{\Delta}} \frac{1}{-p^{2}+2 i \sqrt{s} p \cdot \hat{\mathscr{D}}+s \hat{\Delta}}\right)_{\rho \rho}^{c e}\left(i p_{v}+\hat{\mathscr{D}}_{v}\right)^{e d}\right] .
\end{aligned}
$$


A straightforward expansion with respect to $\sqrt{s}$ then yields

$$
\left\langle b_{\mu}^{a}(t, x)\right\rangle=g_{0}^{2} f^{a b c} \hat{\mathscr{D}}_{\mu}^{b c}(2-D) \int_{0}^{t} d s s^{-D / 2+1} \int \frac{d^{D} p}{(2 \pi)^{D}} e^{-2 p^{2}}\left[\frac{4}{D}+\frac{1}{p^{2}}\left(-1+\frac{2}{D}\right)\right]+O\left(t^{1+\varepsilon}\right),
$$

and this momentum integration identically vanishes for any $D$. This shows that one-loop tadpole diagrams do not contribute to the $O\left(t^{0}\right)$ terms in Eq. (2.J).

Next, we consider one-loop diagrams which arise from the contraction of quantum fields in the last line of Eq. $([2.8)$ by propagators $([2.3)$ and $([2.6)$. A procedure being similar to that led to Eq. (2.15) yields Eq. (3.16) of Ref. [ए0]]; it is

$$
\begin{aligned}
& \left\langle\left. G_{\mu \rho}^{a}(t, x) G_{v \rho}^{a}(t, x)\right|_{O\left(b^{2}\right)}-\left.F_{\mu \rho}^{a}(x) F_{v \rho}^{a}(x)\right|_{O\left(a^{2}\right)}\right\rangle \\
& =2 g_{0}^{2} \int_{0}^{t} d \xi \xi^{-D / 2} \int \frac{d^{D} p}{(2 \pi)^{D}} e^{-2 p^{2}} \\
& \quad \times \operatorname{tr}\left[\xi^{-1} \mathscr{P}_{\mu \alpha, v \delta, \beta \gamma}\left(i p_{\alpha}+\sqrt{\xi} \hat{\mathscr{D}}_{\alpha}\right)\left(e^{4 i \sqrt{\xi} p \cdot \hat{\mathscr{D}}+2 \xi \hat{\Delta}}\right)_{\beta \gamma}\left(i p_{\delta}+\sqrt{\xi} \hat{\mathscr{D}}_{\delta}\right)\right. \\
& \left.+\hat{\mathscr{F}}_{\mu \rho}(x)\left(e^{4 i \sqrt{\xi} p \cdot \hat{\mathscr{D}}+2 \xi \hat{\Delta}}\right)_{\rho v}+\hat{\mathscr{F}}_{v \rho}(x)\left(e^{4 i \sqrt{\xi} p \cdot \hat{\mathscr{D}}+2 \xi \hat{\Delta}}\right)_{\rho \mu}\right],
\end{aligned}
$$

where

$$
\mathscr{P}_{\mu \alpha, v \delta, \beta \gamma} \equiv \delta_{\mu \alpha} \delta_{v \delta} \delta_{\beta \gamma}-\delta_{\mu \alpha} \delta_{v \gamma} \delta_{\beta \delta}-\delta_{\mu \beta} \delta_{v \delta} \delta_{\alpha \gamma}+\delta_{\mu \beta} \delta_{v \gamma} \delta_{\alpha \delta}
$$

We note that, in the present background-gauge-covariant formulation, this compact expression ([.]工) contains all the information equivalent to the tedious diagrammatic expansion computed in Ref. [ए]].

We first compute the second term of Eq. (2.J7):

$$
2 g_{0}^{2} \int_{0}^{t} d \xi \xi^{-D / 2} \int \frac{d^{D} p}{(2 \pi)^{D}} e^{-2 p^{2}} \operatorname{tr}\left[\hat{\mathscr{F}} \mu \rho(x)\left(e^{4 i \sqrt{\xi} p \cdot \hat{\mathscr{D}}+2 \xi \hat{\Delta}}\right)_{\rho v}\right]
$$

The expansion of this expression for $t \rightarrow 0$ is easy because, as noted in Ref. [ए0]], only terms symmetric under $\mu \leftrightarrow v$ contribute by definition. From Eqs. (A1) and (A2) of Ref. [Ш0], we immediately see that the expansion yields

$$
\begin{aligned}
& 8 g_{0}^{2} \int_{0}^{t} d \xi \xi^{-D / 2+1} \int \frac{d^{D} p}{(2 \pi)^{D}} e^{-2 p^{2}} \operatorname{tr}[\hat{\mathscr{F}} \mu \rho(x) \hat{\mathscr{F}} \rho v(x)]+O\left(t^{3-D / 2}\right) \\
& =\frac{g_{0}^{2}}{(4 \pi)^{2}} \frac{(8 \pi t)^{\varepsilon}}{\varepsilon} 2 \operatorname{tr}\left[\hat{\mathscr{F}}(x)^{2}\right]_{\mu \nu}+O\left(t^{1+\varepsilon}\right)
\end{aligned}
$$

The last term in Eq. ([2] gives rise to the same contribution.

The computation of the first term of Eq. (2.17) is somewhat complicated. We first consider the expression without the factor $\mathscr{P}_{\mu \alpha, \nu \delta, \beta \gamma}$;

$$
2 g_{0}^{2} \int_{0}^{t} d \xi \xi^{-D / 2-1} \int \frac{d^{D} p}{(2 \pi)^{D}} e^{-2 p^{2}} \operatorname{tr}\left[\left(i p_{\alpha}+\sqrt{\xi} \hat{\mathscr{D}}_{\alpha}\right)\left(e^{4 i \sqrt{\xi} p \cdot \hat{\mathscr{D}}+2 \xi \hat{\Delta}}\right)_{\beta \gamma}\left(i p_{\delta}+\sqrt{\xi} \hat{\mathscr{D}}_{\delta}\right)\right]
$$


We then use Eqs. (A1)-(A3) of Ref. [प]]. We can neglect $O\left(\xi^{-D / 2}\right)$ terms in the integrand, because these terms give rise to $O\left(t^{-1}\right)$ terms in Eq. (2.17) for $D \rightarrow 4$ which must be absent from gauge invariance. After the expansion, we carry out the momentum integration. Then since

$$
\mathscr{P}_{\mu \alpha, v \delta, \beta \gamma} X_{\alpha \delta} Y_{\beta \gamma}=X_{\mu v} Y_{\rho \rho}-X_{\mu \rho} Y_{\rho v}-X_{\rho v} Y_{\mu \rho}+X_{\rho \rho} Y_{\mu v}
$$

for the combination (2.18), if either $X$ or $Y$ is symmetric under the exchange of indices and the other is anti-symmetric, the product $\mathscr{P}_{\mu \alpha, v \delta, \beta \gamma} X_{\alpha \delta} Y_{\beta \gamma}$ is anti-symmetric under $\mu \leftrightarrow v$. Such term should not contribute to Eq. (2.17). Thus, what we have to retain are only combinations such that both $X$ and $Y$ are symmetric or anti-symmetric under the exchange of indices. This observation greatly simplifies our task. After some calculation, we have for Eq. (2.2]1),

$$
\begin{aligned}
& \frac{g_{0}^{2}}{(4 \pi)^{2}} \frac{(8 \pi t)^{\varepsilon}}{1-\varepsilon / 2} \frac{1}{16 t^{2}} \delta_{\alpha \delta} \delta_{\beta \gamma} \operatorname{dim} G \\
& +\frac{g_{0}^{2}}{(4 \pi)^{2}} \frac{(8 \pi t)^{\varepsilon}}{\varepsilon} \operatorname{tr}\left\{-\delta_{\alpha \delta} \hat{\mathscr{F}}(x)_{\beta \gamma}^{2}-\left[\hat{\mathscr{D}}_{\alpha}, \hat{\mathscr{D}}_{\delta}\right] \hat{\mathscr{F}}(x)_{\beta \gamma}\right. \\
& +\frac{1}{12} \delta_{\beta \gamma}\left[\hat{\mathscr{D}}_{\alpha} \hat{\mathscr{D}}_{\varepsilon} \hat{\mathscr{D}}_{\delta} \hat{\mathscr{D}}_{\varepsilon}+\hat{\mathscr{D}}_{\delta} \hat{\mathscr{D}}_{\varepsilon} \hat{\mathscr{D}}_{\alpha} \hat{\mathscr{D}}_{\varepsilon}-\hat{\mathscr{D}}_{\alpha} \hat{\mathscr{D}}^{2} \hat{\mathscr{D}}_{\delta}-\hat{\mathscr{D}}_{\delta} \hat{\mathscr{D}}^{2} \hat{\mathscr{D}}_{\alpha}\right. \\
& -\hat{\mathscr{D}}_{\varepsilon} \hat{\mathscr{D}}_{\alpha} \hat{\mathscr{D}}_{\delta} \hat{\mathscr{D}}_{\varepsilon}-\hat{\mathscr{D}}_{\varepsilon} \hat{\mathscr{D}}_{\delta} \hat{\mathscr{D}}_{\alpha} \hat{\mathscr{D}}_{\varepsilon}+\hat{\mathscr{D}}_{\varepsilon} \hat{\mathscr{D}}_{\alpha} \hat{\mathscr{D}}_{\varepsilon} \hat{\mathscr{D}}_{\delta}+\hat{\mathscr{D}}_{\varepsilon} \hat{\mathscr{D}}_{\delta} \hat{\mathscr{D}}_{\varepsilon} \hat{\mathscr{D}}_{\alpha} \\
& \left.\left.-\delta_{\alpha \delta} \hat{\mathscr{D}}_{\varepsilon} \hat{\mathscr{D}}_{\varphi} \hat{\mathscr{D}}_{\varepsilon} \hat{\mathscr{D}}_{\varphi}+\delta_{\alpha \delta} \hat{\mathscr{D}}_{\varepsilon} \hat{\mathscr{D}}^{2} \hat{\mathscr{D}}_{\varepsilon}\right]\right\}+O\left(t^{1+\varepsilon}\right) \\
& =\frac{g_{0}^{2}}{(4 \pi)^{2}} \frac{(8 \pi t)^{\varepsilon}}{1-\varepsilon / 2} \frac{1}{16 t^{2}} \delta_{\alpha \delta} \delta_{\beta \gamma} \operatorname{dim} G \\
& +\frac{g_{0}^{2}}{(4 \pi)^{2}} \frac{(8 \pi t)^{\varepsilon}}{\varepsilon} \operatorname{tr}\left[-\delta_{\alpha \delta} \hat{\mathscr{F}}(x)_{\beta \gamma}^{2}-\hat{\mathscr{F}}(x)_{\alpha \delta} \hat{\mathscr{F}}(x)_{\beta \gamma}-\frac{1}{6} \delta_{\beta \gamma} \hat{\mathscr{F}}(x)_{\alpha \delta}^{2}+\frac{1}{24} \delta_{\alpha \delta} \delta_{\beta \gamma} \hat{\mathscr{F}}(x)_{\rho \rho}^{2}\right] \\
& +O\left(t^{1+\varepsilon}\right)
\end{aligned}
$$

where we have repeatedly used the fundamental relation $\left[\hat{\mathscr{D}}_{\mu}, \hat{\mathscr{D}}_{\nu}\right]=\hat{\mathscr{F}}_{\mu \nu}$. Finally, taking the contraction with $\mathscr{P}_{\mu \alpha, v \delta, \beta \gamma}$,

$$
\begin{aligned}
& \frac{g_{0}^{2}}{(4 \pi)^{2}} \frac{1-2 \varepsilon / 3}{1-\varepsilon / 2}(8 \pi t)^{\varepsilon} \frac{3}{8 t^{2}} \operatorname{dim} G \delta_{\mu \nu} \\
& \quad+\frac{g_{0}^{2}}{(4 \pi)^{2}} \frac{(8 \pi t)^{\varepsilon}}{\varepsilon} \operatorname{tr}\left[\left(-\frac{1}{3}+\frac{7}{3} \varepsilon\right) \hat{\mathscr{F}}(x)_{\mu \nu}^{2}+\left(-\frac{11}{12}-\frac{1}{6} \varepsilon\right) \delta_{\mu v} \hat{\mathscr{F}}(x)_{\rho \rho}^{2}\right]+O\left(t^{1+\varepsilon}\right) .
\end{aligned}
$$

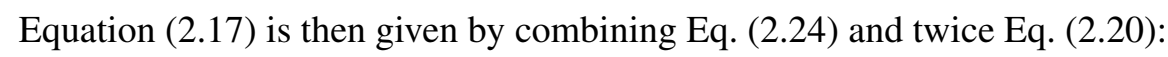

$$
\begin{aligned}
& \frac{g_{0}^{2}}{(4 \pi)^{2}} \frac{1-2 \varepsilon / 3}{1-\varepsilon / 2}(8 \pi t)^{\varepsilon} \frac{3}{8 t^{2}} \operatorname{dim} G \delta_{\mu \nu} \\
& \quad+\frac{g_{0}^{2}}{(4 \pi)^{2}} \frac{(8 \pi t)^{\varepsilon}}{\varepsilon} \operatorname{tr}\left[\left(\frac{11}{3}+\frac{7}{3} \varepsilon\right) \hat{\mathscr{F}}(x)_{\mu \nu}^{2}+\left(-\frac{11}{12}-\frac{1}{6} \varepsilon\right) \delta_{\mu v} \hat{\mathscr{F}}(x)_{\rho \rho}^{2}\right]+O\left(t^{1+\varepsilon}\right) .
\end{aligned}
$$

Finally, we note $\operatorname{tr}\left[\hat{\mathscr{F}}(x)_{\mu \nu}^{2}\right]=C_{2}(G) \hat{F}_{\mu \rho}^{a}(x) \hat{F}_{v \rho}^{a}(x)$. Since we can use the tree-level relations (2.9) in the above one-loop results, we can read off the coefficients in Eq. (2.8) for $\varepsilon \rightarrow 0$ from Eq. ([2.25):

$$
\zeta_{11}(t)=1+\frac{g_{0}^{2}}{(4 \pi)^{2}} C_{2}(G)\left[\frac{11}{3} \varepsilon(t)^{-1}+\frac{7}{3}\right], \quad \zeta_{12}(t)=\frac{g_{0}^{2}}{(4 \pi)^{2}} C_{2}(G)\left[-\frac{11}{12} \varepsilon(t)^{-1}-\frac{1}{6}\right],
$$


where $\varepsilon(t)^{-1} \equiv 1 / \varepsilon+\ln (8 \pi t)$. These coefficients are fundamental for the construction of a lattice energy-momentum tensor in Refs. [U1, [2]]. The present simple calculational scheme revealed that there were errors in the original diagrammatic calculation in Ref. [W] (the diagrams in which the

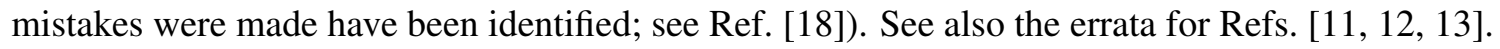

\section{Conclusion}

In the present work, we introduced a background-gauge-covariant gauge fixing in the gradient flow equation. At least in the one-loop order, this formulation allows a very efficient calculational scheme for the small flow time expansion as we illustrated for the composite operator that is relevant to the construction of a lattice energy-momentum tensor. Because of its efficiency, further applications, including two-loop computation of the small flow time expansion, are expected.

I would like to thank Kazuo Fujikawa, Kenji Hieda, and Hiroki Makino for enjoyable discussions. The work of H. S. is supported in part by Grant-in-Aid for Scientific Research 23540330.

\section{References}

[1] M. Lüscher, J. High Energy Phys. 1008, 071 (2010); 1403, 092 (2014) [erratum] [arXiv:1006.4518 [hep-lat]].

[2] M. Lüscher and P. Weisz, J. High Energy Phys. 1102, 051 (2011) [arXiv:1101.0963 [hep-th]].

[3] M. Lüscher, J. High Energy Phys. 1304, 123 (2013) [arXiv:1302.5246 [hep-lat]].

[4] A. Ramos, arXiv:1506.00118 [hep-lat].

[5] G. 't Hooft, Acta Universitatis Wratislaviensis 1, 345 (1976).

[6] B. S. DeWitt, Proc. Quantum Gravity 2, Oxford, 449-487 (1980).

[7] D. G. Boulware, Phys. Rev. D 23, 389 (1981).

[8] L. F. Abbott, Nucl. Phys. B 185, 189 (1981).

[9] S. Ichinose and M. Omote, Nucl. Phys. B 203, 221 (1982).

[10] H. Suzuki, Prog. Theor. Exp. Phys. 2015, 103B03 (2015) [arXiv:1507.02360 [hep-lat]].

[11] H. Suzuki, Prog. Theor. Exp. Phys. 2013, $083 B 03$ (2013); 2015, 079201 (2015) [erratum] [arXiv:1304.0533 [hep-lat]].

[12] H. Makino and H. Suzuki, Prog. Theor. Exp. Phys. 2014, 063B02 (2014); 2015, 079202 (2015) [erratum] [arXiv:1403.4772 [hep-lat]].

[13] M. Asakawa et al. [FlowQCD Collaboration], Phys. Rev. D 90, no. 1, 011501 (2014) [Phys. Rev. D 92, no. 5, 059902 (2015)] [arXiv:1312.7492 [hep-lat]].

[14] K. Fujikawa, Phys. Rev. D 48, 3922 (1993).

[15] H. Makino and H. Suzuki, arXiv:1403.4772v5 [hep-lat].

[16] K. Fujikawa, Phys. Rev. Lett. 42, 1195 (1979).

[17] K. Fujikawa, Phys. Rev. D 21, 2848 (1980); 22, 1499 (1980) [erratum].

[18] H. Suzuki, arXiv:1304.0533v6 [hep-lat]. 